\title{
Developmentally induced changes in transcriptional program alter spatial organization across chromosomes
}

\author{
Jason M. Casolari, ${ }^{1,3}$ Christopher R. Brown, ${ }^{1,3}$ David A. Drubin, ${ }^{1}$ Oliver J. Rando, ${ }^{2}$ \\ and Pamela A. Silver ${ }^{1,4}$ \\ ${ }^{1}$ Department of Systems Biology, Harvard Medical School and The Dana-Farber Cancer Institute, Boston, Massachusetts \\ 02115, USA; ${ }^{2}$ The Bauer Center for Genomics Research, Harvard University, Cambridge, Massachusetts 02138, USA
}

\begin{abstract}
Although the spatial location of genes within the nucleus has been implicated in their transcriptional status, little is known about the dynamics of gene location that accompany large-scale changes in gene expression. The mating of haploid yeast Saccharomyces cerevisiae is accompanied by a large-scale change in transcription and developmental program. We examined changes in nuclear organization that accompany stimulus by the mating pheromone $\alpha$ factor and found that most $\alpha$-factor-induced genes become associated with components of the nuclear envelope. The myosin-like protein Mlp1, which has been implicated in mRNA export, was further shown to exhibit RNA dependence in its association with $\alpha$-factor-induced genes. High-resolution mapping of association of chromosome III with Mlp1 revealed $\alpha$-factor-dependent determinants of nuclear pore association, including origins of replication, specific intergenic regions, and the $3^{\prime}$ ends of transcriptionally activated genes. Taken together, these results reveal RNA- and DNA-dependent determinants of nuclear organization as well as a detailed picture of how an entire chromosome alters its spatial conformation in response to a developmental cue.
\end{abstract}

[Keywords: Nuclear organization; Mlp1; RanGEF; genome polarization; nucleoporin; NPC]

Supplemental material is available at http://www.genesdev.org.

Received February 17, 2005; revised version accepted April 5, 2005.

The nucleus is a complex organelle consisting of nonrandomly positioned chromosomes that are organized into spatially and functionally distinct subdomains. The differential positioning of genes near the nuclear periphery, chromatin domains, or proteinaceous subcompartments suggests that individual loci are exposed to distinct local environments. While such positioning is a fundamental property of genes, the functional significance of this subnuclear organization remains unclear. Several studies in higher eukaryotes have examined the complex organization of the nuclear interior by studying broad yet distinct chromosomal regions such as heterochromatin. For example, in human lymphocytes, the transcriptional regulator Ikaros promotes repression by recruiting target genes into close proximity with heterochromatin (Brown et al. 1997, 1999; Cobb et al. 2000; Cohen et al. 2000). Other groups have focused on studying the dynamics of individual loci and their association with the nuclear periphery. The cystic fibrosis transduc-

\footnotetext{
${ }^{3}$ These authors contributed equally to this work.

${ }^{4}$ Corresponding author.

E-MAIL pamela_silver@dfci.harvard.edu; FAX (617) 632-5103.

Article and publication are at http://www.genesdev.org/cgi/doi/10.1101/ gad.1307205.
}

tion receptor (CFTR) gene and its two nearest neighbors, GASZ and CORTB2, were found to associate with the periphery independently of each other but in direct correlation with their transcriptional state (Zink et al. 2004).

The budding yeast, Saccharomyces cerevisiae, has emerged as a powerful model organism to study nuclear organization as evidence suggests that significant gene regulation occurs at the nuclear periphery. Silenced yeast telomeres are associated with the nuclear periphery via the nuclear pore complex (NPC) and genes localized in proximity to these telomeres are transcriptionally inactive (Gottschling et al. 1990; Gotta et al. 1996; Laroche et al. 1998; Galy et al. 2000; Feuerbach et al. 2002). The NPC also plays a role in maintaining the inactive status of the silent mating-type loci, $H M R$ and HML. The NPC genes NUP60 and NUP145 are required for full repression of the HMR locus, and deletion of the nuclear pore-associated myosin-like protein genes, $M L P 1$ and $M L P 2$, results in the de-repression of a reporter gene at the HML locus (Galy et al. 2000; Feuerbach et al. 2002). Additionally, defects in the silencer sequence of the $H M R$ locus can be artificially suppressed by tethering it to the nuclear membrane while the $H M L$ locus is de-repressed when relocated from its subtelo- 
meric site to a more centromere-proximal location or to the arm of a different chromosome (Stavenhagen and Zakian 1994; Thompson et al. 1994; Maillet et al. 1996; Marcand et al. 1996; Andrulis et al. 1998). Finally, a recent study in $S$. cerevisiae showed that nuclear transport factors and NPC proteins were able to activate a reporter gene by isolating it from silent chromatin (Ishii et al. 2002).

As components of the NPC have been implicated in the proper regulation of transcription, we determined how the entire yeast genome interacts with components of the NPC and transport machinery (Casolari et al. 2004). We made the surprising finding that a subset of transcriptionally active genes was found associated with the nuclear periphery in addition to the expected infrequently transcribed genes. Moreover, when the transcription of genes involved in galactose metabolism was induced, the genes relocated from the nuclear interior to localize at the nuclear pore. Another study has shown that transcriptional activation of the INO1 gene involves its recruitment to the nuclear periphery as well (Brickner and Walter 2004). Taken together, these studies indicate that gene recruitment to the nuclear periphery is a dynamic process that can also play a positive role in gene activation. However, despite the recent advances in describing the interplay between nuclear organization and gene regulation, the basic principles underlying the establishment of nuclear architecture as well as the dynamics of this organization during changes in developmental program remain unknown.

Using genomic location analysis in S. cerevisiae, we have examined how genes interact with components of the NPC after a new transcriptional program is induced by the mating pheromone, $\alpha$ factor. We find that the $\alpha$-factor-induced genes on 13 different chromosomes become associated with the nuclear transport machinery, indicating a global relocalization of newly activated genes to the nuclear periphery. In addition, we find that Mlp1 association with $\alpha$-factor-induced genes is RNase sensitive, indicating that transcription of nascent mRNA leads to association of a subset of loci with the periphery. Moreover, high-resolution mapping of chromosome III localization at the nuclear periphery revealed $\alpha$-factor-dependent determinants of nuclear organization, including origins of replication, intergenic regions, and a bias toward the 3 ' ends of transcriptionally active genes. These data reveal specific determinants of nuclear organization and how an entire chromosome alters its conformation upon induction of a new transcriptional program.

\section{Results}

Induction of $\alpha$-factor-responsive genes leads to changes in nuclear transport-factor association

We wished to determine the extent to which a global change in the transcriptional program of the cell would alter subnuclear dynamics and organization. We therefore chose to examine the mating response of S. cerevi- siae, which involves a pronounced change in the cellular transcriptional program, including induction of matingspecific genes, arrest in G1 of the cell cycle, and gross morphological changes of the plasma membrane and nucleus.

In order to examine gene location following a global transcriptional response, cells were arrested in G1 by treating an exponentially growing culture with $30 \mathrm{nM} \alpha$ factor for $2 \mathrm{~h}$ before formaldehyde fixation. More than $95 \%$ of cells exhibited mating projections, and a G1 arrest was confirmed by FACS (data not shown). Chromatin immunoprecipitated with components of the nuclear transport machinery was examined on whole-genome ORF arrays as previously described (see Materials and Methods; Casolari et al. 2004). These components included the nuclear export factor Xpo1, the nuclear pore protein Nup116, and the myosin-like nuclear poreassociated proteins $\mathrm{Mlp} 1$ and Mlp2. The results of the genomic location analysis for one of these proteins, Mlp1, will be discussed; binding data for Xpo1, Nup116, and Mlp2 were nearly identical to that of Mlp1 and are found in the accompanying Supplemental Material.

Genomic binding profiles generated from cells treated with $\alpha$ factor were analyzed for the enrichment of functional gene classes using FuncAssociate (Berriz et al. 2003). We find that Mlp1 shows enrichment for binding to gene classes induced by mating only in the $\alpha$-factorinduced cells, including genes induced during sexual reproduction and conjugation with cellular fusion (Supplemental Material). Previously published data indicate that 49 genes are induced greater than threefold after a 2-h exposure to $\alpha$ factor, with some genes induced as high as 30-fold (Spellman et al. 1998). We therefore compared the percentile rank of the 49 induced genes in the Mlp1-bound data set with and without $\alpha$ factor (Fig. 1A). Without $\alpha$ factor, the 49 genes are dispersed throughout the data set, indicating no preference for Mlp1 binding to genes from these classes. However, a dramatic change in the binding profile was seen upon addition of $\alpha$ factor, with 35 of the 49 most highly induced genes found in the 90th percentile or greater, indicating a positive correlation between transcriptional activity and binding by Mlp1. Similar results were obtained for Xpo1, Nup116, and Mlp2 (Supplemental Material), demonstrating that transcriptional activation is strongly correlated with binding to the nuclear transport machinery. Furthermore, recruitment of $\alpha$-factor-activated genes to the nuclear periphery occurs on 13 of the 16 yeast chromosomes, indicating that an altered transcriptional program can affect the large-scale organization of the $S$. cerevisiae genome.

The $49 \alpha$-factor-induced genes were analyzed for RanGEF binding with and without $\alpha$ factor, and a disparate binding pattern from that of Mlp1 was observed (Fig. 1B). RanGEF is the intranuclear GTP exchange factor for Ran, is essential for both nuclear transport and chromosome dynamics, and has previously been shown to bind infrequently transcribed genes (Ohtsubo et al. 1987; Aebi et al. 1990; Clark et al. 1991; Forrester et al. 1992; Kadowaki et al. 1994; Azuma and Dasso 2000; Casolari et al. 
Casolari et al.

Figure 1. Correlation among genome occupancy, transcriptional activation, and peripheral localization. All genes in the genomic occupancy profiles of Mlp1 and RanGEF/Prp20 were assigned a percentile rank ranging from $0 \%$ (unbound) to $100 \%$ (highest binding). The distributions of the 49 genes induced threefold or more upon $\alpha$-factor treatment (Spellman et al. 1998) within the Mlp1 $(A)$ and RanGEF/Prp20 $(B)$ data sets are shown for untreated cells (white bars) and cells treated with 30 $\mathrm{nM} \alpha$ factor for $2 \mathrm{~h}$ (black bars). A bin size of $10 \%$ was used. Combined FISH/IF of the FIG2 locus in cells that were untreated $(C)$ and stimulated with $30 \mathrm{nM} \alpha$ factor for $2 \mathrm{~h}(D)$. The FIG2 probe is visualized as a single green spot (see yellow arrow) with nuclear pore staining in red. The outline of each stimulated cell is shown in white, with the shmoo projection highlighted by a white arrow. (E) Mlp1 genomic location analysis was carried out as in $A$ except that cells were fixed for only 5 min plus or minus RNase prior to immunoprecipitation $(F)$. The distribution of the 49 genes induced threefold or more upon $\alpha$-factor treatment are shown for untreated cells (white bars) and cells treated with $30 \mathrm{nM} \alpha$ factor for $2 \mathrm{~h}$ (black bars).
A

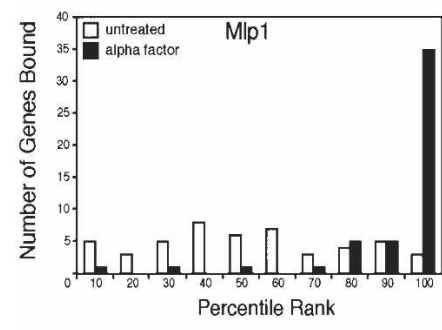

C

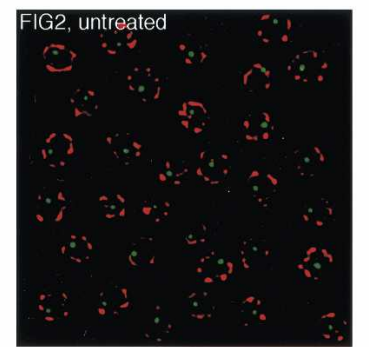

$E$

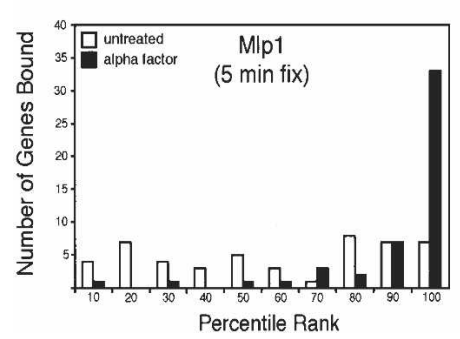

B

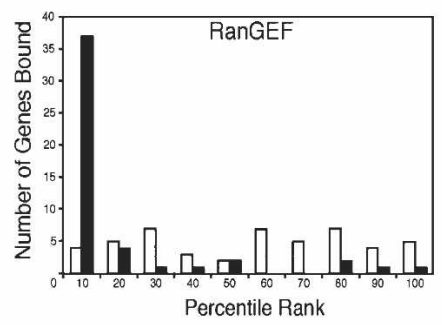

D

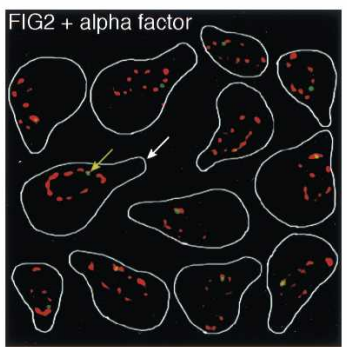

$\mathbf{F}$

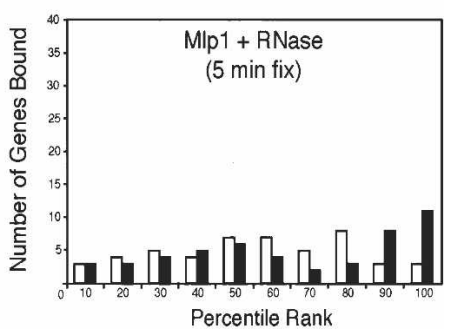

2004). In the presence of $\alpha$ factor, 37 of the 49 genes are found in the 10th percentile or lower of the bound population, while in the absence of $\alpha$ factor they are distributed throughout the data set. Interestingly, the only significant class found to be enriched in the RanGEF data set was sporulation genes. This binding profile illustrates the disparate binding properties of RanGEF, compared with Mlp1, since it has concomitantly dissociated from active $\alpha$-factor-induced genes and acquired binding to the transcriptionally inactive sporulation genes.

\section{$\alpha$-Factor treatment leads to gene recruitment to the nuclear periphery}

We performed fluorescent in situ hybridization (FISH) coupled with immunofluorescence (IF) in order to confirm that the changes in protein association we observed using genomic location analysis reflected recruitment of the activated genes to the nuclear periphery. FIG2, a gene induced $\sim 3.5$-fold by the addition of $\alpha$ factor (Spellman et al. 1998; Guo et al. 2000), encodes an adhesin required for maintaining cell wall integrity during mating (Erdman et al. 1998; Guo et al. 2000). A 5-kb DNA probe was designed to hybridize to FIG2 and was visualized by incorporating digoxigenin-dUTP and staining with a FITC-conjugated anti-DIG antibody. NPCs were visualized with an anti-Myc antibody in Nup116-Myc tagged strains. In untreated cells, the FIG2 locus is found predominantly localized in the nuclear interior with only $27 \%$ of cells showing localization at the nuclear periphery (Fig. 1C). Upon induction with $\alpha$ factor, the FIG2 signal overlaps with the nuclear periphery in the majority (64\%) of cells. Interestingly, FIG2 was found to localize to the side of the nucleus that was closest to the mating projection in $93 \%$ of cells (Fig. 1D). Indeed, the Fig2 protein has also been shown to localize solely to the shmoo mating projection with exclusion from the rest of the plasma membrane (Guo et al. 2000).

\section{Mlp1 association with $\alpha$-factor-induced genes is RNA dependent}

A number of previous studies have implicated Mlp1 in mRNA export; Mlp1 is known to bind to the hnRNP and mRNA export factor, Nab2 (Green et al. 2003). In addition, deletion of Mlp1 causes leakage of unspliced premRNAs to the cytoplasm (Galy et al. 2004), while overexpression leads to accumulation of poly $\mathrm{A}(+)$ mRNA in the nucleus (Kosova et al. 2000). Given that Mlp1 has direct links to mRNA export and shows association with $\alpha$-factor-responsive genes in a transcription-dependent manner, we sought to determine if Mlp1 association with these genes might occur via the transcribed mRNA.

To achieve this, genomic location analysis of Mlp1 was repeated on chromatin from cells that were lightly fixed and treated with RNase A and T1 during the im- 
munoprecipitation before application to whole-genome ORF arrays. As shown in Figure 1E, the shorter fixation time used for thorough RNase digestion does not affect the ability of Mlp1 to bind to $\alpha$-factor-responsive genes. However, after treatment with RNase, we see a dramatic decrease in the association of Mlp1 with the transcriptionally induced genes (Fig. 1F). Mlp1 binding to subtelomeric genes in both conditions is not affected (Supplemental Material), demonstrating that Mlp1 association with some loci is not dependent upon RNA.

High-resolution mapping of chromosome III binding reveals determinants of association with the nuclear periphery

Since association of genes with the NPC varies with transcriptional status and local chromatin organization (Galy et al. 2000; Feuerbach et al. 2002; Casolari et al. 2004), we sought to identify the sequence determinants responsible for this association. To achieve this goal, we performed genomic localization of Mlp1 using a highresolution, tiled microarray of $S$. cerevisiae chromosome III (see Materials and Methods). Mlp1 was chosen for high-resolution genomic location analysis based upon its previously identified roles in $H M L$ and $H M R$ silencing as well as its ties to mRNA export. Mlp1-bound chromatin was prepared as described from cells grown in rich media (YEPD) (see Materials and Methods) and competitively hybridized to tiled arrays comprised of 50-bp probes at 20-bp intervals across the entire length of chromosome III, allowing for the precise localization of Mlp1 to portions of the chromosome.

Using full-genome ORF arrays, we find that Mlp1 binding to chromosome III is comprised of transcriptionally active genes such as PGK1 (110 mRNAs/h) and PMP1 (38.7 mRNAs/h), infrequently transcribed genes such as CDC39 (0.8 mRNAs/h) and YCL044C (0.2 mRNAs/h), as well as the silent mating type loci $H M R$ and $H M L$ (Fig. 2A; Holstege et al. 1998; Casolari et al. 2004). As shown in Figure 2C, origins of replication, termed autonomously replicating sequences (ARS) in yeast, are present in the Mlp1-bound data set. All of these ARS elements are adjacent to regions that have been previously localized to the nuclear periphery, such as $H M L, H M R$, and the centromere (Jin et al. 1998; Laroche et al. 2000).

Strikingly, we found that Mlp1 binding along chromosome III also appears to be the result of association with both ORF and intergenic sequences (Fig. 2D,E). Association with some ORFs, such as RER1, is due to binding at the $3^{\prime}$ end of the ORF in addition to intergenic binding downstream (Fig. 2F). Other Mlp1-bound sites show extensive binding across an entire intergenic region, such as the 864-bp region separating TAF2 and YCR043C, without overlap into the ORFs themselves (Fig. 2F).

Use of high-resolution tiled arrays to examine gene association with Mlp1 also affords an even higher level of refinement in identification of binding sites. For example, the Mlp1 binding to PMP1 shown using fullgenome ORF arrays (Fig. 2A) can be narrowed to a 140-bp sequence within the 379-bp intergenic region separating PMP1 and YCR025C (see Supplemental Material). In addition, the Mlp1 localization at $H M L$ and $H M R$ can be refined to include specific enrichment at ARS elements and intergenic regions at these loci (Fig. 2F).

Change in the transcription program causes alteration in the determinants of binding at the nuclear periphery

Since treatment of yeast with $\alpha$ factor causes a dramatic change in the loci associated with the nuclear periphery, we sought to further characterize the global and local determinants of Mlp1 association with genes using tiled arrays and chromosome III as a model. As shown in Figure 3A, several regions of the chromosome change in their association with Mlp1 upon $\alpha$-factor treatment as indicated by upward (gain in binding) and downward (loss of binding) arrows. Notably, the three $\alpha$-factor-induced genes on chromosome III, KAR4 (7.7-fold), FUS1 (5.5-fold), and FIG2 (3.5-fold) (Spellman et al. 1998), all gain association with Mlp1 after $\alpha$-factor treatment as predicted from the ORF arrays. Additionally, the $H M R$ and $H M L$ proximal ARS elements bound by Mlp1 before treatment remain bound, although binding to the centromere proximal ARS308 and ARS309 is lost (Fig. 3C). Surprisingly, genes not induced by $\alpha$ factor, including PDI1, GBP2, YCP4, PHO87, CPR4, and SSK22, also gained association with Mlp1 upon $\alpha$-factor treatment. Binding of PDI1, GBP2, and YCP4 may reflect the constitutively high expression of these genes (Holstege et al. 1998; Spellman et al. 1998). Alternatively, binding to these genes may be a consequence of the semiperiodic association of Mlp1 with chromosome III, which will be discussed later.

In addition to the change of Mlp1 binding to transcriptionally activated loci, local changes in the pattern of Mlp1 occupancy occur. As shown in Figure 4A and B, binding of Mlp1 to PGK1 is not significantly altered after $\alpha$-factor treatment; PGK1 is highly transcribed in both conditions (Holstege et al. 1998; Spellman et al. 1998). In contrast, there is a marked relocation of Mlp1 from the ARS304-adjacent position in untreated cells to binding within the KAR4 ORF after transcriptional induction (Fig. 4C,D). Mlp1 exhibits only background occupancy in the FUS1 and FIG2 regions before exposure to $\alpha$ factor (Fig. 4E,G), demonstrating minor localized binding to the FIG2-adjacent ARS316. However, Mlp1 binding to FUS1 and FIG2 is strongly increased upon induction of the mating pathway, with significant binding throughout the two genes. Moving average graphs demonstrate that Mlp1 occupancy at FUS1 is relatively constant throughout the ORF with peaks at the middle and 3 ' end of the gene (Fig. 4F), while binding to FIG2 shows a steady increase that peaks just downstream of the ORF (Fig. $4 \mathrm{H}$ ).

\section{Discussion}

A number of previous studies have connected localization of genes at the nuclear periphery with their proper 
Casolari et al.

Figure 2. Mlp1 occupancy on chromosome III using whole-genome ORF and high-resolution tiled arrays. (A) Genes bound by Mlp1 (IP/WCE > 1.0 and $p$-value $\leq 0.05$ ) on whole-genome ORF arrays (Casolari et al. 2004) are displayed (blue) on a spatial representation of $S$. cerevisiae chromosome III (gray bar). (B) High-resolution mapping of Mlp1-bound probes (see Materials and Methods) on tiled arrays is shown on a representation of chromosome III (gray bar). Data having a standard deviation $>2.0$ and a moving average $>2.0$ are shown. Binding to probes within ARS elements (red), ORFs (blue), and intergenic sequences (green) are depicted with colored bars on a spatial representation of the chromosome. $(C-E)$ Significantly bound probes were mapped to the corresponding ARSes, ORFs, and intergenic sequences and are labeled on representations of chromosome III. (F) Mlp1 binding to regions of chromosome III is displayed with a moving average of nine adjacent probes. Black arrows indicate the direction of transcription for each ORF. Gray arrows represent ORFs considered dubious by the Saccharomyces Genome Database, and open boxes represent transposon sequences.
A

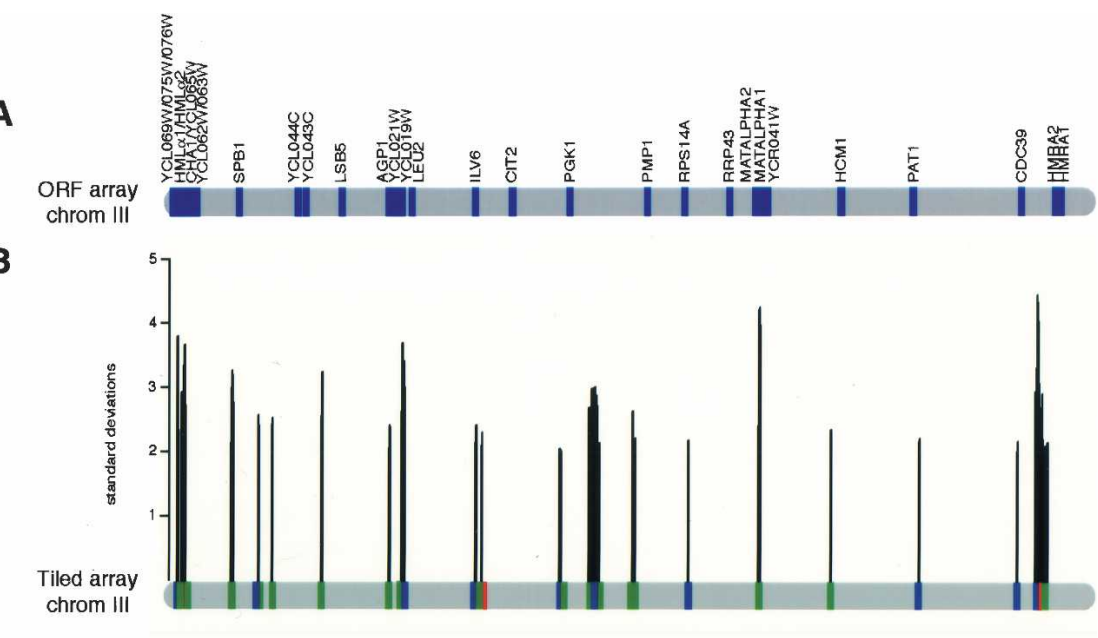

B

C ARS

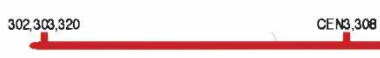

317,318

D ORF

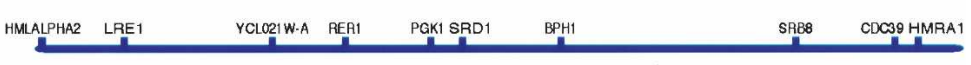

E
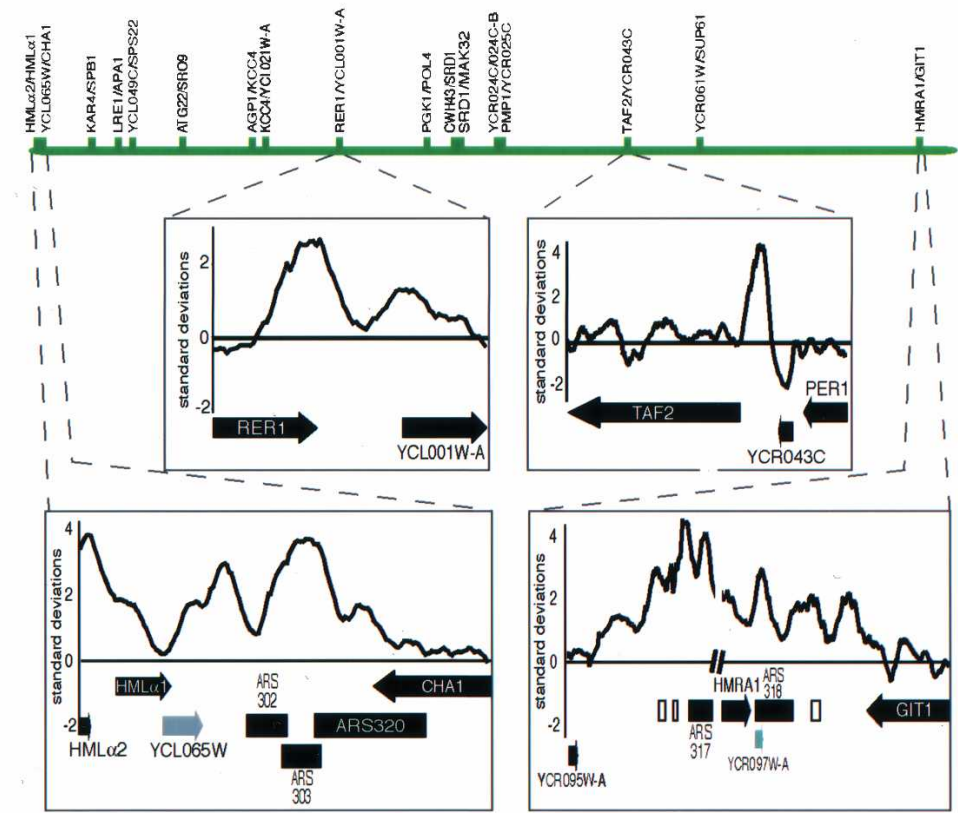

transcriptional regulation (Gotta and Gasser 1996; Gotta et al. 1996; Andrulis et al. 1998, 2002; Galy et al. 2000; Feuerbach et al. 2002; Hediger et al. 2002; Ishii et al. 2002). While the nuclear periphery has generally been viewed as a transcriptionally repressive zone, work by Ishii et al. (2002) demonstrated that components of the nuclear transport machinery exhibit strong boundary activities, permitting activation of a reporter gene by isolating it from a silent chromatin environment. Furthermore, previous work from our laboratory and others showed that the GAL and INO1 genes relocalize to the nuclear periphery upon transcriptional induction (Brickner and Walter 2004; Casolari et al. 2004), suggesting that the nuclear periphery is not prohibitive of transcriptional activity.
In order to address the generality of these findings, as well as to identify the local and global determinants of chromosome association with the nuclear periphery, we mapped the genome-wide binding pattern of the nuclear pore-associated protein, Mlp1, before and after the initiation of the mating transcriptional program. These experiments have led to several major conclusions. First, $\alpha$-factor-induced genes from 13 of the 16 yeast chromosomes bind the nuclear transport machinery, indicating a global rearrangement of the genome after the induction of a new transcriptional program. Second, $\alpha$-factor treatment leads to FIG2 localization at the region of the nuclear periphery closest to the shmoo mating projection, indicating that the genome of $S$. cerevisiae exhibits polarization. Third, association of Mlp1 with induced 
A

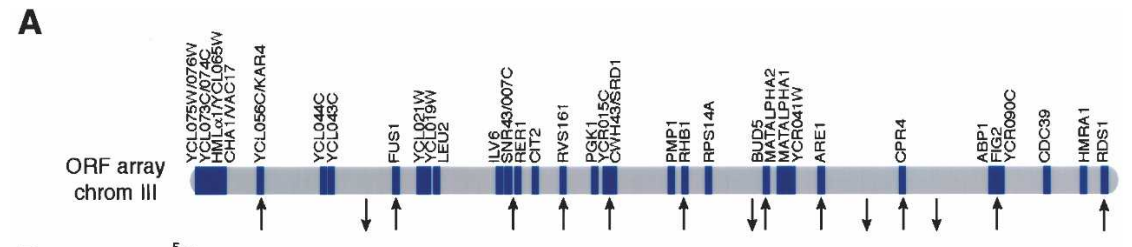

B

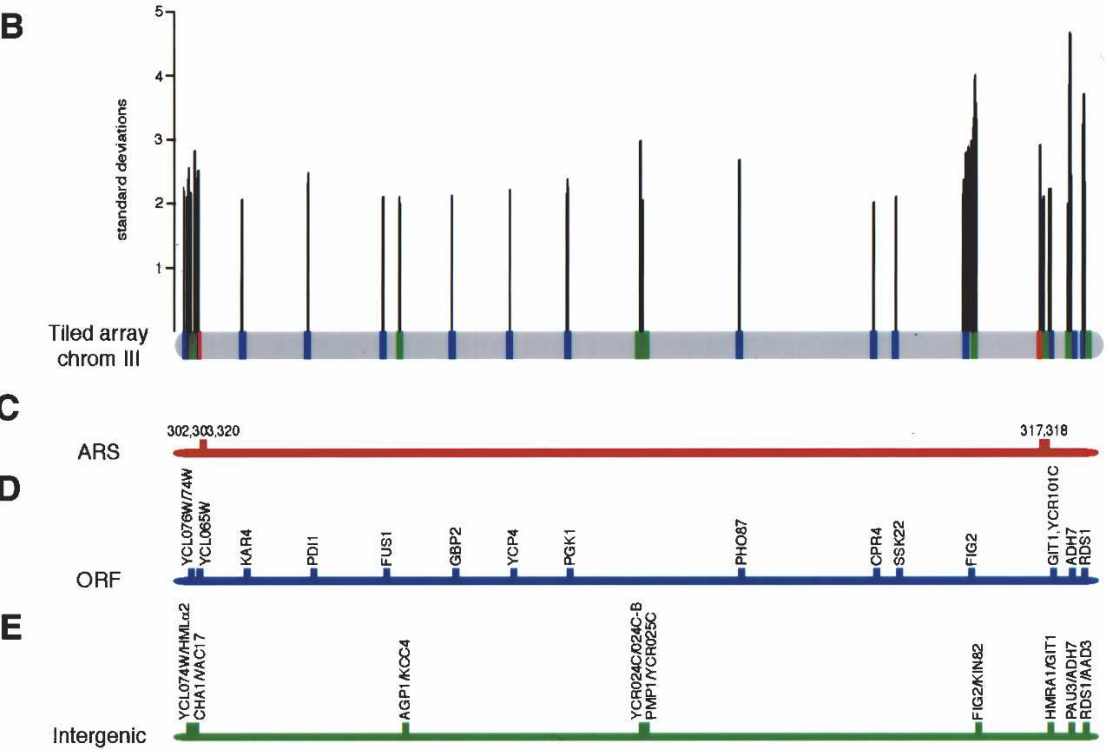

Figure 3. Mlp1 occupancy on chromosome III after stimulation with $\alpha$ factor. Genes bound by Mlp1 on whole-genome ORF arrays $(A)$ and tiled arrays $(B)$ after stimulation with $\alpha$ factor are displayed on a spatial representation of $S$. cerevisiae chromosome III (gray bar). $\alpha$-Factor-induced changes in Mlp1 binding to ORF arrays, relative to untreated cells, are represented with upward (gain in binding) and downward (loss of binding) arrows. $(C-E)$ Binding to ARS, ORF, and intergenic regions are displayed as in Figure 2. genes is RNA dependent while binding to subtelomeric genes is not, establishing RNA and DNA as independent determinants in establishing nuclear architecture. Finally, Mlp1 associates with distinct genomic elements, including transcriptionally activated ORFs, intergenic regions, the silent mating-type loci, and ARS sequences, indicating that a number of sequence determinants contribute to localization at the nuclear periphery.

\section{Transcriptional activity and nuclear organization}

Mlp1 binding to $\alpha$-factor-induced genes is consistent with our previous finding that GAL genes relocate to the nuclear periphery upon induction (Casolari et al. 2004). Indeed, FIG2 was found to localize at the nuclear periphery with a nearly identical frequency to the induced $G A L$ locus (Casolari et al. 2004) as well as INO1 (Brickner and Walter 2004). We believe that this increase reflects the dynamic nature of genomic loci within the yeast nucleus (Heun et al. 2001b), with certain transcriptionally active loci spending more time at the nuclear periphery.

We also find that Mlp1 binding at transcriptionally activated genes is pronounced with a bias toward the $3^{\prime}$ end. In both untreated and $\alpha$-factor-stimulated cells, Mlp1 shows occupancy at PGK1, the gene encoding 3 -phosphoglycerate kinase and one of the most highly transcribed genes in yeast (Holstege et al. 1998). Highresolution mapping of Mlp1-chromosome III association reveals robust binding across the entire PGK1 coding region, with the greatest enrichment at the $3^{\prime}$ end. Mlp1 enrichment at the $3^{\prime}$ end of FUS1 and FIG2 is also ob- served upon transcriptional induction. Notably, Mlp1 binding to FUS1 and other $\alpha$-factor-induced genes, including FUS2, ASG7, and KAR3, is drastically decreased when chromatin is treated with RNase, indicating that Mlp1 association with these genes occurs through an RNA intermediate. Indeed, one possible explanation for the existence of intergenic Mlp1-binding sites is that these regions reflect unknown areas of transcription.

Mlp1 has previously been shown to genetically interact with mutant alleles of the mRNA export factors YRA1 and NAB2 as well as physically interacting with the Nab2 protein (Green et al. 2003; Vinciguerra et al. 2005). As such, our results suggest that Mlp1 association with RNA-binding proteins such as Nab2 might facilitate association of transcriptionally active genes with the NPC. Our data also support our previously proposed model (Casolari et al. 2004), in which transcriptionally active loci are recruited to the pore in order to facilitate direct entry of the nascent mRNA into the processing and export pathways.

Intriguingly, FIG2 recruitment to the nuclear periphery appears to be polarized, with nearly all cells showing localization of the gene at the side of the nucleus closest to the shmoo projection. The spindle pole body (SPB) is asymmetrically distributed within the nuclear envelope and found in the region nearest the emerging bud in vegetatively growing cells (Baba et al. 1989; Yang et al. 1989; Snyder et al. 1991). Previous work has also suggested that centromeres should lie near the SPB (Yang et al. 1989 ), yet FIG2 is proximal to the right telomere of chromosome III, indicating that the polarized FIG2 localization likely reflects a different role. 
Casolari et al.

A

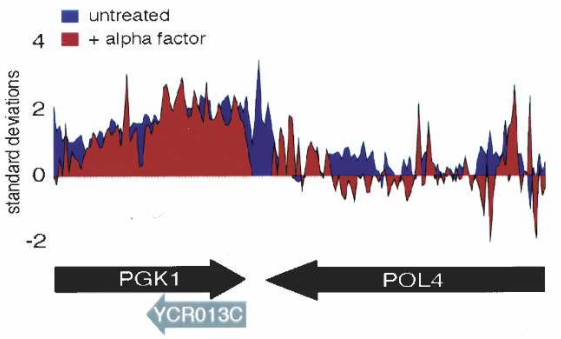

C

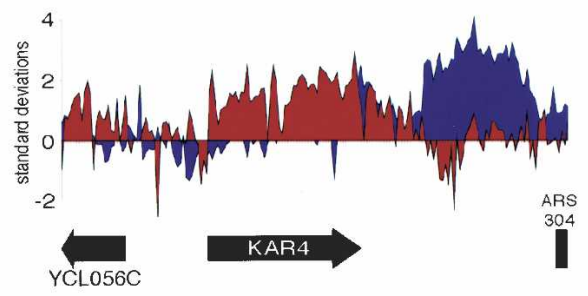

E
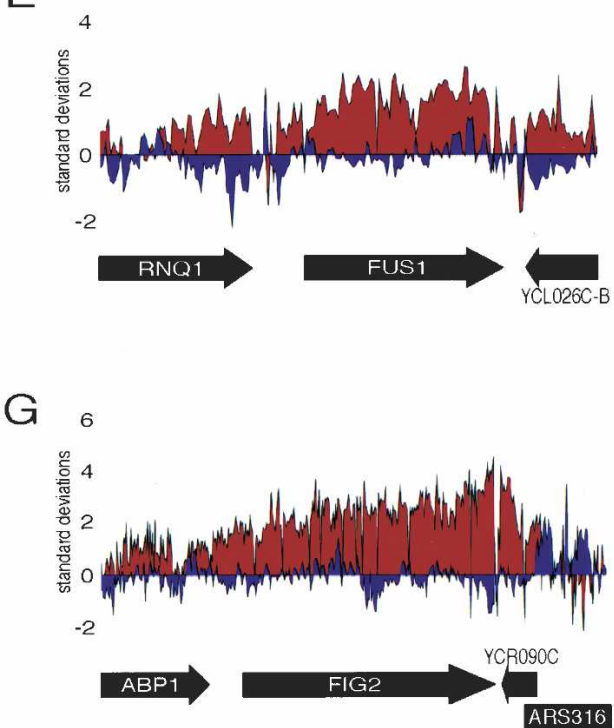

B

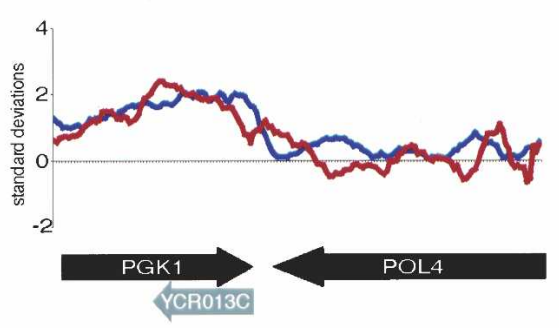

D

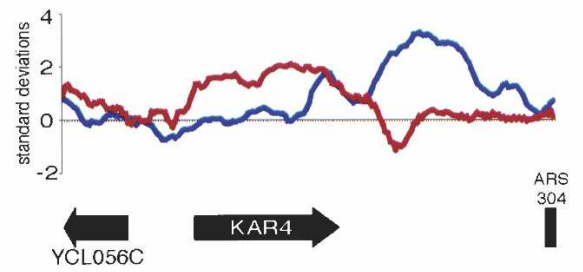

F

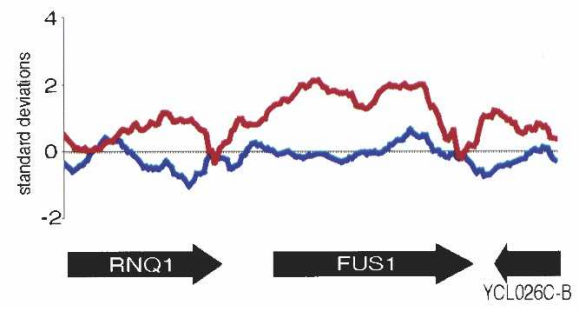

$\mathrm{H}$

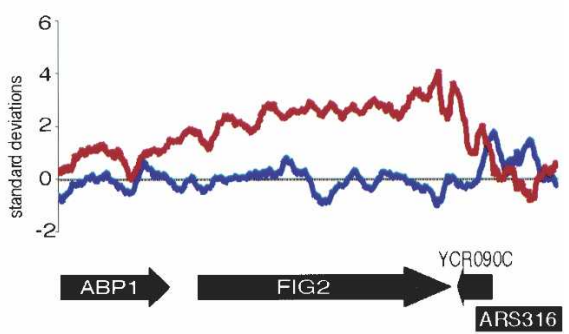

Figure 4. Local changes in Mlp1 occupancy upon $\alpha$-factor stimulation of transcription. Mlp1 binding to transcriptionally active regions are shown. Standard deviations for individual probes $(A, C, E, G)$ as well as a moving average of binding for nine adjacent probes $(B, D, F, H)$ are shown. ORFs are displayed as in Figure 2.

While it is not clear if the asymmetric recruitment of FIG2 precedes or follows formation of the mating projection, it is tempting to suggest that a mechanism exists to favor directional export of the FIG2 mRNA from the nucleus. Indeed, the Fig2 protein is expressed exclusively during mating and is found only in the portion of the plasma membrane where the shmoo mating projection is formed (Guo et al. 2000). In Chlamydomonas reinhardtii, nuclear asymmetry has also been observed; NPCs were observed to occupy a region of the nucleus nearest the site of cytoplasmic transcript accumulation (Colon-Ramos et al. 2003). Our observation of a genomic locus that is also asymmetrically localized within the nucleus extends these observations and suggests that not only is the nucleus polarized but the genome is as well. These observations suggest that the establishment of nuclear asymmetry is a general phenomenon that may enhance proper mRNA exit and localization.

\section{Binding to sites of replication}

The timing of origin of replication firing in S. cerevisiae has been determined (Raghuraman et al. 2001), and previous studies have demonstrated enrichment for some of these ARS elements within different regions of the nucleus. In cells synchronized in the G1 phase of the cell cycle, late-firing origins tended to be enriched at the nuclear periphery, while early-firing origins localized 
preferentially to the nuclear interior. During the $S$ and G2 phases of the cell cycle, late-origins of replication tended to lose their association with the nuclear periphery (Heun et al. 2001a). As Mlp1 shows perinuclear localization and is associated with the NPC (Strambio-deCastillia et al. 1999; Galy et al. 2000, 2004; Kosova et al. 2000), we expect that only late-firing origins of replication be enriched for Mlp1 binding in our G1-arrested, $\alpha$-factor-treated cells. As demonstrated using chromosome III tiled arrays, the most robust binding of ARS elements was observed at the silent mating-type loci, $H M R$ and $H M L$, which are each flanked with several late-firing origins of replication and are known to associate with the nuclear periphery (Andrulis et al. 1998, 2002; Feuerbach et al. 2002).

Interestingly, the $\alpha$-factor-induced genes FUS1, FIG2, and KAR4 are all closely linked to origins of replication, separated by $\sim 1.2 \mathrm{~kb}, \sim 400 \mathrm{bp}$, and $\sim 1.5 \mathrm{~kb}$, respectively. If association with the nuclear periphery plays a role in prohibiting the firing of origins of replication in yeast (Heun et al. 2001a), then one could propose that the linear arrangement of the $\alpha$-factor-responsive genes on chromosome III has been selected for in evolution. Cells that have sensed the presence of mating pheromone would induce transcription of FUS1, FIG2, and KAR4, recruit the genes to the nuclear periphery, and concomitantly prohibit firing of the adjacent ARS elements to ensure that cells do not enter S phase. It is also worth noting that ARS elements are adjacent to $\alpha$-factor-induced genes only on chromosome III; as chromosome III is the yeast sex chromosome, this may reflect a unique synergy between the processes of transcription, ARS firing, and nuclear organization.

\section{Chromosome conformational change}

Several attempts have been made to understand the dynamics of whole chromosomes in living cells (for review, see Parada et al. 2004). These previous studies have relied upon broad, low-resolution views of whole chromosome localization and narrow, high-resolution studies of individual loci and their localization within the nucleus. Using genomic location analysis of the perinuclear Mlp1 protein, whole-genome ORF arrays, and high-resolution tiled arrays of chromosome III, we have obtained detailed local and global information regarding the organization of the yeast genome. On a gross level, we have observed that a change in the developmental program of the cell causes a dramatic shift in the loci associated with the nuclear periphery, including recruitment of genes from 13 of the 16 yeast chromosomes. On a higher-resolution scale, we were also able to observe local changes in binding at the nuclear periphery.

As a number of local changes in chromosome III association occur after $\alpha$-factor stimulation, we sought to determine where the most pronounced changes in Mlp1 binding took place. In order to assess the overall change in the conformation of chromosome III relative to the nuclear periphery, we took advantage of the experimentally determined value for interlocus cross-linking fre- quency on chromosome III. Work by Dekker et al. (2002) has demonstrated that the cross-linking frequency between chromosome III loci is maximal for sites separated by $8.5-10 \mathrm{~kb}$. These data suggest that loci separated by this distance are sufficiently far apart to allow looping of the intervening chromatin, and thus, loci at the two extreme ends are close enough in three dimensions to allow interlocus cross-linking.

Using this information, we determined regions of semicontinuous association with Mlp1 and thus the nuclear periphery. Mlp1-bound loci separated by $<8.5 \mathrm{~kb}$ were considered to be part of a stretch of semicontinuous binding, whereas bound loci separated by $>8.5 \mathrm{~kb}$ were considered far enough apart to allow interlocus "looping." We feel that the use of the 8.5 -kb interval is conservative as two genomic loci do not necessarily need to be near each other in three-dimensional space, as assumed by their ability to be cross-linked, in order to contact the nuclear periphery as separate entities. As a result, the use of this interval minimized the importance of local changes in binding and allowed us to discern those regions of chromosome III showing the most dramatic changes in association with the periphery.

As shown in Figure 5, we have been able to derive a population average model of chromosome III conformation relative to the nuclear periphery and its change upon initiation of the mating transcriptional program. On a gross level, we observed extensive distances between the contacts of Mlp1 with chromosome III, though the endpoints of these looped regions remained constant in several instances between the two different conditions. Semicontinuous Mlp1 binding was found to be limited to the chromosomal ends and is comprised of the mating type loci as well as the adjacent subtelomeric regions. The areas of greatest change between untreated and $\alpha$-factor-stimulated cells are highlighted in red in Figure $5 \mathrm{~B}$ and can be generally mapped to a region stretching from 28 to $120 \mathrm{~kb}$ (region 2) and another region extending from 180 to $285 \mathrm{~kb}$ (region 4).

The mapping of the Mlp1-associated regions is highly similar to a previous description of two large GC-rich domains present on chromosome III that exhibit extensive interlocus cross-linking and stretch from 25 to 100 $\mathrm{kb}$ and 190 to $280 \mathrm{~kb}$. Dekker et al. (2002) hypothesize that the large degree of curvature predicted to exist in these GC-rich domains might arise from some "intrinsic bias in fiber path" or from an external source such as association with the nuclear envelope. Our data bear out these predictions and further suggest that regions 2 and 4 are particularly amenable to changes in association with the nuclear periphery upon alteration in the transcriptional program. The dynamic contacts between Mlp1 and regions 2 and 4 may represent an inherent bias toward peripheral localization of the resident genes or, alternatively, a more stringent requirement for the maintenance of peripheral localization for the genes residing in regions 1, 3, and 5. Given that the nuclear peripheryassociated silent mating-type loci, centromere, and telomeres (Gotta et al. 1996; Jin et al. 1998; Heun et al. 2001a,b; Tham et al. 2001) reside in regions 1, 3, and 5, 
Figure 5. Global changes in Mlp1 occupancy reflect conformational changes in chromosome III relative to the nuclear periphery. $(A)$ Mlp1 binding on chromosome III in untreated and $\alpha$-factorstimulated cells is shown. Binding to sites separated by $<8.5 \mathrm{~kb}$ is considered semicontinuous and represented with a horizontal line at the nuclear periphery. Binding gaps of $>8.5 \mathrm{~kb}$ are represented by a loop extending into the nuclear interior that is proportional to the distance between Mlp1-binding sites. Regions where data are unavailable are represented by breaks in the line. $(B)$ The regions of binding that are held constant under the two conditions, stretching from 0 to $\sim 28 \mathrm{~kb}$ (region 1), $\sim 120$ to $180 \mathrm{~kb}$ (region 3), and $\sim 285$ to $300 \mathrm{~kb}$ (region 5), are displayed in black. Regions exhibiting pronounced changes in binding pattern between the two conditions (regions 2 and 4) are displayed in red.
A

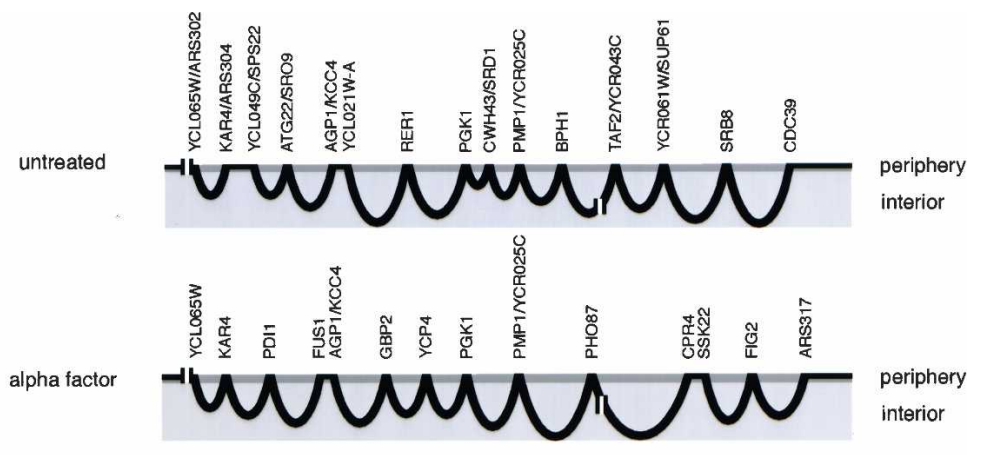

B

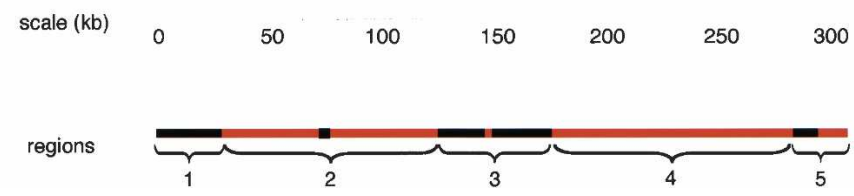

we hypothesize that the latter of these two models is more likely to be correct.

\section{Materials and methods \\ Strains and growth conditions}

The haploid yeast strains of the background PSY2156/Z1256 (MATa, ade2-1, trp1-1, can1-100, leu2-3, 112, his3-11, 15, ura3) (Ren et al. 2000) previously used (Casolari et al. 2004) were transformed with a PCR-amplified cassette to replace the endogenous BAR1 locus with a KanMX4 insert in order to enhance $\alpha$-factor sensitivity. The following strains were created to allow for $\alpha$-factor stimulation of cells prior to chromatin immunoprecipitation and microarray analysis: nup116:Myc bar1:KanMX4 (PSY3270), xpo1:Myc bar1:KanMX4 (PSY3271), mlp1:Myc bar1:KanMX4 (PSY3289), mlp2:Myc bar1:KanMX4 (PSY3290), and prp20:Myc bar1:KanMX4 (PSY3291). Yeast strains were grown to an $\mathrm{OD}_{600}$ of 0.2 in YEPD, and $\alpha$-factor peptide (US Biological) was added to a concentration of $50 \mathrm{ng} / \mathrm{mL}(30 \mathrm{nM})$. After $120 \mathrm{~min}$ of stimulation, cultures were fixed with $1 \%$ formaldehyde for $20 \mathrm{~min}$ at $30^{\circ} \mathrm{C}$ before washing and collection of cells.

\section{Genomic location analysis and microarray hybridization}

ORF arrays The experimental procedure for chromatin immunoprecipitation and hybridization to ORF microarrays (University Health Networks) was performed as previously described (Casolari et al. 2004) unless otherwise stated. Sample preparation for the RNase-treated samples was based on a protocol from Abruzzi et al. (2004) and differed from our chromatin immunoprecipitation in the following ways: (1) Cells were fixed in $1 \%$ formaldehyde for $5 \mathrm{~min}$ before washing and collection of cells, and (2) $11 \mathrm{U}$ of RNase A and $450 \mathrm{U}$ of RNase T1 (Ambion) were added to the IP samples and allowed to incubate for $30 \mathrm{~min}$ at room temperature before the immunoprecipitation was performed.

Tiled arrays Samples for tiled microarrays differed from ORF array preparation in two ways: (1) Chromatin was extensively sonicated to produce DNA with an average size of 100-200 bp, and (2) $\sim 2 \mu \mathrm{g}$ of immunoprecipitated DNA (IP) and whole-cell extract (WCE) were labeled with Cy5-dUTP or Cy3-dUTP (PerkinElmer) before competitive hybridization to microarrays. The tiled arrays used are comprised of 50-base oligonucleotides spanning S. cerevisiae chromosome III (O.J. Rando, unpubl.) staggered every $20 \mathrm{bp}$. Tiled microarray hybridization was performed for $4 \mathrm{~h}$ at $55^{\circ} \mathrm{C}$ in buffer containing $3.4 \times \mathrm{SSC}$ and $0.3 \%$ SDS. Arrays were washed sequentially with $1 \times$ SSC/0.03\% SDS, $0.2 \times$ SSC, and $0.05 \times$ SSC. Microarrays were then scanned, and fluorescence intensities quantified with an Axon Genepix 4000B scanner and software.

\section{Combined FISH/IF}

A 4.6-kb fragment spanning the coding sequence of FIG2 was amplified from genomic DNA by using the following primer sequences: 5'-CTCATTTGCGTCATTAGGTCTGA-3' and 5'TCGTCGTTACTGATGGAATTACAGTT-3'. The digoxigenindUTP derivatized FIG2 probe was resuspended in hybridization solution $(50 \%$ formamide, $10 \%$ dextran sulfate, $2 \times \mathrm{SSC})$ to a final concentration of $10 \mathrm{ng} / \mathrm{mL}$. Combined FISH/IF was performed using a modified protocol based on a previously described technique (Gotta et al. 1999; Casolari et al. 2004). PSY3270 cells (nup116:Myc bar1:KanMX4) were grown in rich media with or without $30 \mathrm{nM} \alpha$ factor (US Biological) at $30^{\circ} \mathrm{C}$ to a density of $1 \times 10^{7}$ cells $/ \mathrm{mL}$ and then fixed in $4 \%$ paraformaldehyde before spheroplasting to prevent nuclear spreading. The monoclonal anti-myc antibody, 9E10 (Santa Cruz Biotechnology), was used at a 1:200 dilution. Preabsorbed Alexa Fluor 568 goat anti-mouse (Molecular Probes) and sheep anti-digoxigeninFITC (Roche) were used at 1:1000 and 1:50 respectively. Cells were imaged using a Nikon TE2000U inverted microscope with PerkinElmer ultraview spinning disk confocal. A stringent requirement for complete overlap with the NPC was used to score FIG2 region localization at the nuclear periphery.

\section{Microarray data analysis}

ORF microarrays were scanned and analyzed as previously described (Casolari et al. 2004). IP/WCE ratios for four (untreated) and five ( $\alpha$-factor treated) tiled array data sets were normalized around zero for all probes on the arrays before the median value was taken. In order to minimize the influence of cross-hybridization to probes representing different regions of chromosome III, moving averages were calculated for a sliding window of 
nine adjacent probes. Only loci exhibiting standard deviations $>2.0$ and a moving average $>2.0$ were considered bound. This cutoff eliminated a number of dubious single-probe binding calls and produced data sets that were similar to those obtained using whole ORF arrays.

\section{Acknowledgments}

We thank P. Grosu, R. Gali, and the Bauer Center for Genomics Research (Harvard University) for help with microarray analysis; A. Davol and M. Manley for help with data analysis scripts; A. McKee and M. Yu for helpful discussions about experimental design; and K. Auld, N. Gilks, J. Hurt, C. Kennedy, J. Way, and $\mathrm{M}$. Yu for comments on the manuscript. This work was supported by a training grant from the National Eye Institute to J.M.C., a training grant from the U.S. National Institutes of Health to C.R.B. and D.A.D., funding from the Bauer Center for Genomics Research at Harvard University to O.J.R., and grants from the U.S. National Institutes of Health to P.A.S.

\section{References}

Abruzzi, K.C., Lacadie, S., and Rosbash, M. 2004. Biochemical analysis of TREX complex recruitment to intronless and intron-containing yeast genes. EMBO J. 23: 2620-2631.

Aebi, M., Clark, M.W., Vijayraghavan, U., and Abelson, J. 1990. A yeast mutant, PRP20, altered in mRNA metabolism and maintenance of the nuclear structure, is defective in a gene homologous to the human gene RCC1 which is involved in the control of chromosome condensation. Mol. Gen. Genet. 224: 72-80.

Andrulis, E.D., Neiman, A.M., Zappulla, D.C., and Sternglanz, R. 1998. Perinuclear localization of chromatin facilitates transcriptional silencing. Nature 394: 592-595.

Andrulis, E.D., Zappulla, D.C., Ansari, A., Perrod, S., Laiosa, C.V., Gartenberg, M.R., and Sternglanz, R. 2002. Esc1, a nuclear periphery protein required for Sir4-based plasmid anchoring and partitioning. Mol. Cell. Biol. 22: 8292-8301.

Azuma, Y. and Dasso, M. 2000. The role of Ran in nuclear function. Curr. Opin. Cell Biol. 12: 302-307.

Baba, M., Baba, N., Ohsumi, Y., Kanaya, K., and Osumi. M. 1989. Three-dimensional analysis of morphogenesis induced by mating pheromone $\alpha$ factor in Saccharomyces cerevisiae. J. Cell. Sci. 94 (pt 2): 207-216.

Berriz, G.F., King, O.D., Bryant, B., Sander, C., and Roth, F.R. 2003. Characterizing gene sets with FuncAssociate. Bioinformatics 19: 2502-2504.

Brickner, J.H. and Walter, P. 2004. Gene recruitment of the activated INO1 locus to the nuclear membrane. PLOS Biol. 2: E342.

Brown, K.E., Guest, S.S., Smale, S.T., Hahm, K., Merkenschlager, M., and Fisher, A.G. 1997. Association of transcriptionally silent genes with Ikaros complexes at centromeric heterochromatin. Cell 91: 845-854.

Brown, K.E., Baxter, J., Graf, D., Merkenschlager, M., and Fisher, A.G. 1999. Dynamic repositioning of genes in the nucleus of lymphocytes preparing for cell division. Mol. Cell 3: 207217.

Casolari, J.M., Brown, C.R., Komili, S., West, J., Hieronymus, H., and Silver, P.A. 2004. Genome-wide localization of the nuclear transport machinery couples transcriptional status and nuclear organization. Cell 117: 427-439.

Clark, K.L., Ohtsubo, M., Nishimoto, T., Goebl, M., and Sprague Jr., G.F 1991. The yeast SRM1 protein and human RCC1 protein share analogous functions. Cell Regul. 2: 781-792.

Cobb, B.S., Morales-Alcelay, S., Kleiger, G., Brown, K.E., Fisher, A.G., and Smale, S.T. 2000. Targeting of Ikaros to pericentromeric heterochromatin by direct DNA binding. Genes \& Dev. 14: 2146-2160.

Cohen, B.A., Mitra, R.D., Hughes, J.D., and Church, G.M. 2000. A computational analysis of whole-genome expression data reveals chromosomal domains of gene expression. Nat. Genet. 26: 183-186.

Colon-Ramos, D.A., Salisbury, J.L., Sanders, M.A., Shenoy, S.M., Singer, R.H., and Garcia-Blanco, M.A. 2003. Asymmetric distribution of nuclear pore complexes and the cytoplasmic localization of $\beta 2$-tubulin mRNA in Chlamydomonas reinhardtii. Dev. Cell 4: 941-952.

Dekker, J., Rippe, K., Dekker, M., and Kleckner, N. 2002. Capturing chromosome conformation. Science 295: 1306-1311.

Erdman, S., Lin, L., Malczynski, M., and Snyder, M. 1998. Pheromone-regulated genes required for yeast mating differentiation. J. Cell. Biol. 140: 461-483.

Feuerbach, F., Galy, V., Trelles-Sticken, E., Fromont-Racine, M., Jacquier, A., Gilson, E., Olivo-Marin, J.C., Scherthan, H., and Nehrbass, U. 2002. Nuclear architecture and spatial positioning help establish transcriptional states of telomeres in yeast. Nat. Cell. Biol. 4: 214-221.

Forrester, W., Stutz, F., Rosbash, M., and Wickens, M. 1992. Defects in mRNA 3 '-end formation, transcription initiation, and mRNA transport associated with the yeast mutation prp20: Possible coupling of mRNA processing and chromatin structure. Genes \& Dev. 6: 1914-1926.

Galy, V., Olivo-Marin, J.C., Scherthan, H., Doye, V., Rascalou, N., and Nehrbass, U. 2000. Nuclear pore complexes in the organization of silent telomeric chromatin. Nature 403: $108-112$.

Galy, V., Gadal, O., Fromont-Racine, M., Romano, A., Jacquier, A., and Nehrbass, U. 2004. Nuclear retention of unspliced mRNAs in yeast is mediated by perinuclear Mlp1. Cell 116: 63-73.

Gotta, M. and Gasser, S.M. 1996. Nuclear organization and transcriptional silencing in yeast. Experientia 52: 1136-1147.

Gotta, M., Laroche, T., Formenton, A., Maillet, L., Scherthan, H., and Gasser, S.M. 1996. The clustering of telomeres and colocalization with Rap1, Sir3, and Sir4 proteins in wild-type Saccharomyces cerevisiae. J. Cell. Biol. 134: 1349-1363.

Gotta, M., Laroche, T., and Gasser, S.M. 1999. Analysis of nuclear organization in Saccharomyces cerevisiae. Methods Enzymol. 304: 663-672.

Gottschling, D.E., Aparicio, O.M., Billington, B.L., and Zakian, V.A. 1990. Position effect at S. cerevisiae telomeres: Reversible repression of Pol II transcription. Cell 63: 751-762.

Green, D.M., Johnson, C.P., Hagan, H., and Corbett, A.H. 2003. The C-terminal domain of myosin-like protein 1 (Mlplp) is a docking site for heterogeneous nuclear ribonucleoproteins that are required for mRNA export. Proc. Natl. Acad. Sci. 100: 1010-1015.

Guo, B., Styles, C.A., Feng, Q., and Fink, G.R. 2000. A Saccharomyces gene family involved in invasive growth, cell-cell adhesion, and mating. Proc. Natl. Acad. Sci. 97: 1215812163.

Hediger, F., Neumann, F.R., Van Houwe, G., Dubrana, K., and Gasser, S.M. 2002. Live imaging of telomeres: yKu and Sir proteins define redundant telomere-anchoring pathways in yeast. Curr. Biol. 12: 2076-2089.

Heun, P., Laroche, T., Raghuraman, M.K., and Gasser, S.M. 2001a. The positioning and dynamics of origins of replication in the budding yeast nucleus. J. Cell. Biol. 152: 385-400.

Heun, P., Laroche, T., Shimada, K., Furrer, P., and Gasser, S.M. 
Casolari et al.

2001b. Chromosome dynamics in the yeast interphase nucleus. Science 294: 2181-2186.

Holstege, F.C., Jennings, E.G., Wyrick, J.J., Lee, T.I., Hengartner, C.J., Green, M.R., Golub, T.R, Lander, E.S., and Young, R.A. 1998. Dissecting the regulatory circuitry of a eukaryotic genome. Cell 95: 717-728.

Ishii, K., Arib, G., Lin, C., Van Houwe, G., and Laemmli, U.K. 2002. Chromatin boundaries in budding yeast: The nuclear pore connection. Cell 109: 551-562.

Jin, Q., Trelles-Sticken, E., Scherthan, H., and Loidl, J. 1998. Yeast nuclei display prominent centromere clustering that is reduced in nondividing cells and in meiotic prophase. J. Cell. Biol. 141: 21-29.

Kadowaki, T., Chen, S., Hitomi, M., Jacobs, E., Kumagai, C., Liang, S., Schneiter, R., Singleton, D., Wisniewska, J., and Tartakoff, A.M. 1994. Isolation and characterization of Saccharomyces cerevisiae mRNA transport-defective (mtr) mutants. J. Cell. Biol. 126: 649-659.

Kosova, B., Pante, N., Rollenhagen, C., Podtelejnikov, A., Mann, M., Aebi, U., and Hurt, E. 2000. Mlp2p, a component of nuclear pore attached intranuclear filaments, associates with nic96p. J. Biol. Chem. 275: 343-350.

Laroche, T., Martin, S.G., Gotta, M., Gorham, H.C., Pryde, F.E., Louis, E.J., and Gasser, S.M. 1998. Mutation of yeast Ku genes disrupts the subnuclear organization of telomeres. Curr. Biol. 8: 653-656.

Laroche, T., Martin, S.G., Tsai-Pflugfelder, M., and Gasser, S.M. 2000. The dynamics of yeast telomeres and silencing proteins through the cell cycle. J. Struct. Biol. 129: 159-174.

Maillet, L., Boscheron, C., Gotta, M., Marcand, S., Gilson, E., and Gasser, S.M. 1996. Evidence for silencing compartments within the yeast nucleus: A role for telomere proximity and Sir protein concentration in silencer-mediated repression. Genes \& Dev. 10: 1796-1811.

Marcand, S., Buck, S.W., Moretti, P., Gilson, E., and Shore, D. 1996. Silencing of genes at nontelomeric sites in yeast is controlled by sequestration of silencing factors at telomeres by Rap 1 protein. Genes \& Dev. 10: 1297-1309.

Ohtsubo, M., Kai, R., Furuno, N., Sekiguchi, T., Sekiguchi, M., Hayashida, H., Kuma, K., Miyata, T., Fukushige, S., Murotsu, T., et al. 1987. Isolation and characterization of the active cDNA of the human cell cycle gene (RCC1) involved in the regulation of onset of chromosome condensation. Genes \& Dev. 1: 585-593.

Parada, L.A., Sotiriou, S., and Misteli, T. 2004. Spatial genome organization. Exp. Cell. Res. 296: 64-70.

Raghuraman, M.K., Winzeler, E.A., Collingwood, D., Hunt, S., Wodicka, L., Conway, A., Lockhart, D.J., Davis, R.W., Brewer, B.J., and Fangman, W.L. 2001. Replication dynamics of the yeast genome. Science 294: 115-121.

Ren, B., Robert, F., Wyrick, J.J., Aparicio, O., Jennings, E.G., Simon, I., Zeitlinger, J., Schreiber, J., Hannett, N., Kanin, E., et al. 2000. Genome-wide location and function of DNA binding proteins. Science 290: 2306-2309.

Snyder, M., Gehrung, S., and Page, B.D. 1991. Studies concerning the temporal and genetic control of cell polarity in Saccharomyces cerevisiae. J. Cell. Biol. 114: 515-532.

Spellman, P.T., Sherlock, G., Zhang, M.Q., Iyer, V.R., Anders, K., Eisen, M.B., Brown, P.O., Botstein, D., and Futcher, B. 1998. Comprehensive identification of cell cycle-regulated genes of the yeast Saccharomyces cerevisiae by microarray hybridization. Mol. Biol. Cell. 9: 3273-3297.

Stavenhagen, J.B. and Zakian, V.A. 1994. Internal tracts of telomeric DNA act as silencers in Saccharomyces cerevisiae. Genes \& Dev. 8: 1411-1422.

Strambio-de-Castillia, C., Blobel, G., and Rout, M.P. 1999. Pro- teins connecting the nuclear pore complex with the nuclear interior. J. Cell. Biol. 144: 839-855.

Tham, W.H., Wyithe, J.S., Ko Ferrigno, P., Silver, P.A., and Zakian, V.A. 2001. Localization of yeast telomeres to the nuclear periphery is separable from transcriptional repression and telomere stability functions. Mol. Cell 8: 189-199.

Thompson, J.S., Johnson, L.M., and Grunstein, M. 1994. Specific repression of the yeast silent mating locus HMR by an adjacent telomere. Mol. Cell. Biol. 14: 446-455.

Vinciguerra, P., Iglesias, N., Camblong, J., Zenklusen, D., and Stutz, F. 2005. Perinuclear Mlp proteins downregulate gene expression in response to a defect in mRNA export. EMBO $J$. 24: $813-823$.

Yang, C.H., Lambie, E.J., Hardin, J., Craft, J., and Snyder, M. 1989. Higher order structure is present in the yeast nucleus: Autoantibody probes demonstrate that the nucleolus lies opposite the spindle pole body. Chromosoma 98: 123-128.

Zink, D., Amaral, M.D., Englmann, A., Lang, S., Clarke, L.A., Rudolph, C., Alt, F., Luther, K., Braz, C., Sadoni, N., et al. 2004. Transcription-dependent spatial arrangements of CFTR and adjacent genes in human cell nuclei. J. Cell. Biol. 166: $815-825$. 


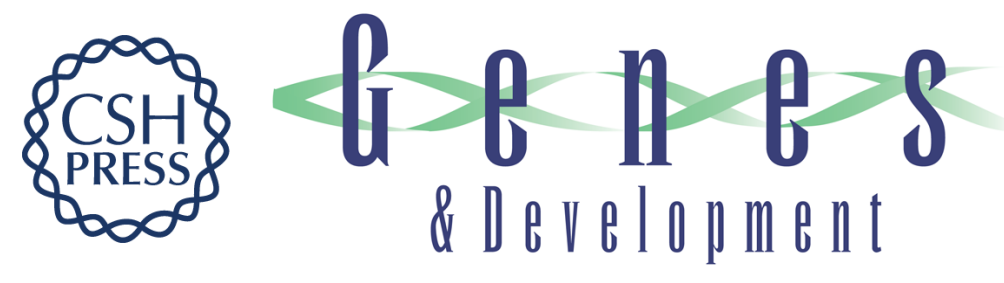

\section{Developmentally induced changes in transcriptional program alter spatial organization across chromosomes}

Jason M. Casolari, Christopher R. Brown, David A. Drubin, et al.

Genes Dev. 2005, 19:

Access the most recent version at doi:10.1101/gad.1307205

Supplemental http://genesdev.cshlp.org/content/suppl/2005/09/26/19.10.1188.DC1
Material

References This article cites 51 articles, 26 of which can be accessed free at:

http://genesdev.cshlp.org/content/19/10/1188.full.html\#ref-list-1

License

Email Alerting

Receive free email alerts when new articles cite this article - sign up in the box at the top

Service

right corner of the article or click here.

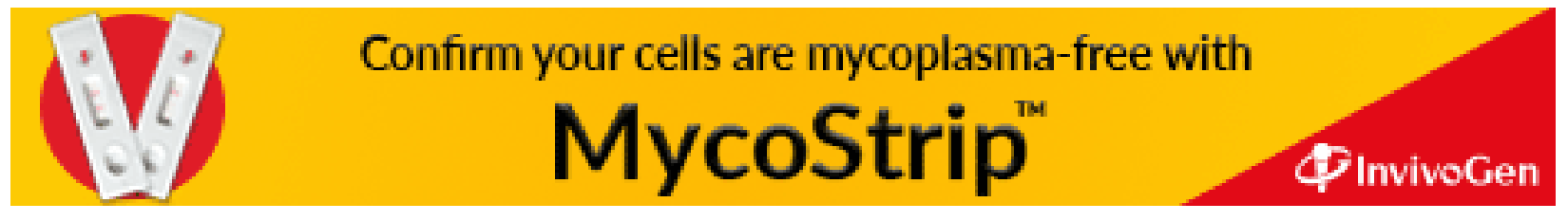

